\title{
Enhanced 5G contender based flat top weighted window communication systems
}

\author{
Omar Abdulkareem Mahmood ${ }^{1}$, Yousif I. Hammadi ${ }^{2}$ \\ ${ }^{1}$ Department of Communications Engineering, College of Engineering, University of Diyala, Baqubah, Iraq \\ ${ }^{2}$ Department of Medical Instruments Engineering Techniques, Bilad Alrafidain University College, Diyala, Baqubah, Iraq
}

\begin{tabular}{l} 
Article Info \\
\hline Article history: \\
Received Aug 28, 2021 \\
Revised Nov 19, 2021 \\
Accepted Dec 31, 2021 \\
\hline
\end{tabular}

\section{Keywords:}

$5 \mathrm{G}$

Flat top weighted window F-OFDM

Out of band emission

Time-frequency localization Windowed-sinc filters

\begin{abstract}
To create a new transmission phenomenon, the availability of good data rates, the development of the internet of things (IoT), and different machine type communications (MTC) developed the ability to converse without synchronization, or the need for routing overhead in the form of synchronization overhead, while using mixed legacy fourth generation (4G) systems, based on orthogonal frequency division multiplexing, cannot meet these requirements (OFDM). Many waveform alternatives for OFDM have been proposed, including FBMC, GFDM, UFMC, and filtering-OFDM, in order to meet the criteria of the next generation system F-OFDM. As a consequence, a revolutionary filter based on a flat top weighted window is described in this work, where simulation results indicate that the suggested method outperforms prior designs in regard to spectral adeptness increased substantially acrros the deminish in the required side-information as overhead of the synchronization process. The trasitional F-OFDM uses rootraised-cosine filter. However, in this paper, novel filter design was proposed, whis is simple and real-time construction capability. This new filter is built around legacy filters, where the new filter is a combination of different filters to make use of various advantages of the old filters and get rid of the disadvangages. The power spectral density (PSD), $-157.2 \mathrm{dBW} / \mathrm{Hz}$, which is outstanding CP-OFDM-PSD, $-50 \mathrm{dBW} / \mathrm{Hz}$.
\end{abstract}

This is an open access article under the CC BY-SA license.

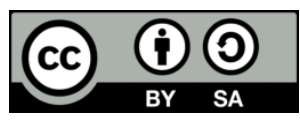

\section{Corresponding Author:}

Yousif I. Hammadi

Department of Medical Instruments Engineering Techniques, Bilad Alrafidain University College

Ba'aqubah 32001, Diyala, Iraq

Email: yousif.ibrahim.hammadi@gmail.com

\section{INTRODUCTION}

Mostly with advancement of technology, fresh demands for information transfer have constituted an unusual challenge for the cutting-edge cell infrastructure. Aside from the standard telecom advantages of cell phones, the current new generation, fifth generation (5G) [1], project is likely to strengthen traffic load that are in particular distinct from the previous counterparts, for illustration, machine type communication (MTC), internet of things (IoT), which seems to be using really quite tiny information transmission as discussed in [2] besides the streaming of different multimedia information [3]. Using adjustable waveforms is a crucial process for dealing with the upcoming challenges, which are shown by Banelli et al. [4]. A primary need of waveform composition in modern generation, $5 \mathrm{G}$, designs is to aid irregular delivery with the particular objective of keeping a staying away from the significant overhead of synchronization signaling required by large endpoints [5], [6]. The cyclic prefix-orthogonal frequency division multiplexing (CPOFDM) modulation and access technology that was used in fourth generation (4G) networks [7]; nevertheless, OFDM can only fulfill the aforementioned requirement since orthogonality among subcarriers 
can't be maintained in irregular or asynchronously broadcasting. As a result, unattractive adjacent-channelinterference (ACI) will result from out-of-band (OOB) output [8].

To put it otherwise, the synchronization overhead is going to cause latency to grow and consume more power; nevertheless, MTC will be battery-powered, and batteries need to survive for a long period [9]. Additionally, regardless of the asynchronous transmission, 5G pulses have a time-domain localization characteristic, which is essential to meet latency and short message delivery requirements [6]. Due to this, filtered signals are a consideration in the $5 \mathrm{G}$ waveforms. Omission-free transmission is possible with filtered waveforms, which cuts down on OOB emissions. Filtered waveforms may be divided into three categories: whole band, subcarrier, and subband. Filterbank multicarrier (FBMC) [10] beats standard CP-OFDM, which utilizes offset-quadrature-amplitude-modulation (OQAM) [11]-[13], whereas generalized frequency division multiplexing (GFDM) [11] is another subcarrier filtering-based waveform. Unfortunately, despite the impressive results, the extended appendage of the filter time-domain-response reduces processing time (latency), where this latency is a critical attribute in modern communication, 5G, and platforms [14]. Bellanger et al. [10] provided a prominent example of previously reported remedies in the context of the large tails, however the latency had worsened. Additionally, FBMC suffers from a deterioration in terms of orthogonality, though results in inter-symbol-interference [9], [13], [15].

In order to compensate the reasons listed above, some intelligent, creative filtered waveforms, built around subbands, successfully developed. The technique configured in order to achieve subband filtering, in which an individual subband having several subcarriers accessible for propagation. In other words, because of the broader filter bandwidth, the filter's impulse response will be smaller than it would be in the case of per subcarrier filter functioning. According to LTE designs, each subband is made up of 12 subcarriers, which are referred to as resource blocks (RB), which is why resource block filtered OFDM was created [16], [17]. The universal filtered multicarrier (UFMC), which corresponds to the subband based filtering group, was also proposed as a contender for 5G [6], [9], [14], [18]. The filter frequency band may be extended further so that the entire range of the conveyed subbands is filtered in one go. That really is, just one filter will be used at the sender. All of this output waveform is known as filtered-OFDM (F-OFDM) [19], [20]. This article will solely cover F-OFDM.

Several numerologies must be used in $5 \mathrm{G}$ to serve multiple MTC structures. These signal components incorporate subcarrier-spacings, changeable cyclic-prefix, signal propagations required time, and so on. Additionally, it is critical to loosen the coordination between the transmitter and the endpoints where heavy signaling burden would be accessible for data transfer. To meet these criteria, the output waveform ought to be versatile enough to be adjusted based on the kind of communication. The CP-OFDM is rectangular-pulse structured in time- and sinc in frequency-domain, respectively. However, sinc-technique's disadvantage is that its sidelobes fade as little by little as $1 /$ frequency. Thus, the sidelobes obstruct frequency localisation, resulting in the loss of concurrence with neighboring services [20]. Likewise, one disadvantage of sinc-sidelobes is the necessity for precise synchronization under both freqiemcu-and-time. As indicated at the commencement of this section, several types of waveforms have been found in the literature to loosen the timing constraints. However, the primary goal of achieving asynchronous communication is to reduce OOB emission [13], [21], [22]. F-OFDM has gained some traction in the literature; in [20], the research introduced a signal that reduces the extended tail of legacy $4 \mathrm{G}$ signals, CP-OFDM, as well as an asynchronous number of co system employing F-OFDM.

Meanwhile, CP-OFDM employed pulse structuring to improve frequency stability, but the suggested F-OFDM inhibits OOB leakage, resulting in improved stability. owing to significant OOB of conventional legacy OFDM signals, protection frequency-distance is mandatory, resulting in a known spectrum inefficiency. Approximately $10 \%$ of the available bandwidth in 4G LTE networks is utilised as guard band [23]. Several techniques were presented in order to accomplish the stability. It is necessary to utilize a different pulse shape in the frequency domain in order to avoid using the sinc-function. For example, during signal processing filter creation, Hanning windowing, for example, is a restriction of the architype filter by windowing its impulse response, is employed in some applications [20]. So, the waveform that will be used must have higher frequency-range competence, temporal and frequencies-stability, and orthogonality amongst subcarriers. Regrettably, as per Gabor's hypothesis [24], we will not be able to achieve all of these characteristics simultaneously. Thus, the signal must be constructed in a thoughtful manner, achieving a reasonable trade in terms of both timing and frequency localization, as well as an increase in transmission capacity over CP-OFDM while maintaining orthogonality. Just use the same method as [20], thus, using dissimilar windowing-function, namely the Von Hann window, [25] discovered the suggested F-OFDM surpasses FBMC and UFMC signals in terms of throughput and efficiency.

Specifically, filtering is required to reduce the OOB emission to a regulated amount in order to overcome disturbance between subsequent subbands. Additional information on F-OFDM was provided in [26], from which a realistic site examinations were performed and demonstrated novel-F-OFDM beats CPOFDM. Wu et al. [26] demonstrates that asynchronous subbands may be utilized with nearby subband 
interfering disregarded. Nonetheless, F-OFDM allows for the employment of bandwidth, which results in the relaxation of more subcarriers for usable information transfer and, as a result, greater transmission capacity [19]. Ku and Armada compared two distinct numerologies, namely MTC and conventional audio data, and the findings demonstrated the F-capacity OFDM's to handle diverse numerologies [27]. Additional field study was run in [21], and it was demonstrated that a more over 7\% increase in data rate was obtained with little interaction between neighboring subbands. The distinction between the two field experiments is that [26] only conducted uplink communication, whereas [21] only did downlink communication. Another proposed approach for reducing OOB generation was explored in [22], in which windowing-filtering or filtering-windowing is a goal that has been reached depending on the position of the filter and/or window. As a result, the design technique will be demonstrated in the next part, along with the introduction of new windows. This article is structured as; section 2 discusses filter design based on the employment of windowing and finite impulse response filter and proposes different F-OFDM development who have used windows that were not in the literary works, however, the novel F-OFDM will be built around flat top window-based filtering approach, additionally, section 4 provides modeling findings and discussion, and section 5 puts the concluding remarks.

\section{METHOD}

For better time and frequency localisation to increase system throughput, F-OFDM effectiveness is heavily dependent on filter design. Methods such as the windowed-sinc approach [28] are straightforward to use, methodical, and may be implemented online. As a matter of fact, this filter design approach has a number of clearly defined windows [29], [30]. This can be described mathematically as:

$$
g_{n}(n)=g_{r}(n) \cdot w(n)
$$

Where $g_{r}(n)$ is the filter's necessary time-domain response. It is also possible to utilize an optimal low-passfilter sinc response [31].

Following the windowing process, the resulting filter must be moved in the frequency domain to the middle of the designated spectrum. Due to the obvious Gibbs-issue [31], the window-functionality of a sincfilter in time, hence this operation, introduces ripple in band's border spectrums. Conversely, the ripple frequency can indeed be removed if the allotted spectrum is prolonged for just few subcarriers at the transformation boundaries; in this way, the filter passes frequencies as straight as feasible for the assigned subband while still beginning its roll-off at the subband's intended boundaries. That would be to state, design process is critical, and as such, the filter design approach should be prudent in terms of selecting an acceptable window function. As a result, reduced $\mathrm{OOB}$ emission can be attained, resulting in substantial improvements in time and frequency localization. Mostly as result, frequency range efficiency can be improved by generating additional bands which could be used for valuable information, in contrast to conventional CP-OFDM. As a direct consequence, data throughput increases. There are several window types described in the literature [29], [30], each with its own set of pros and drawbacks. For example, the most basic type is the rectangular-window. The primary disadvantage of the rectangle function is its poor frequency domain localization, as a result, it is not approved for use in $5 \mathrm{G}$ networks.

Taher et al [30] implemented different novel filters to enhance the F-OFDM waveform contender based on the original theoretical background explained in [32], where it was discussed in [32] that many windows have been generated using two of more different windows, in other words by mixing/combining distinct windows. For example, triangular/Bartlett-window was generated by convolving two rectangular windows [30], [32]. The objective was to create a window with a thinner main lobe width and extremely low sidelobe depths. In [30], the authors completely designed different windows, based on the theory of [32], such as Hanning-Hanning window, which is the convolution of the Hanning-window by itself. Thus, it is mathematically can be describe as:

$$
w_{\text {new }}(n)=w_{1}(n) \cdot w_{2}(n)
$$

where $w_{1}$ is the first window and $w_{2}$ denotes the second window. In this work, it is proposed to design a new window that has smaller-width of the main lobe and less sidelobe levels compared to other windows in the literature.

The comprehensive review of the state-of-the-art, it can be said that flat-top weighted-window has extremely low passband ripple, less than $0.01 \mathrm{~dB}$, and it is designed originally to be used for the purpose of calibration [33]. Flat-top weighted window can be described mathematically using four-cosine terms, 


$$
w_{F T W}(n)=\tau_{0}-\tau_{1} \cos \left[\frac{2 \pi}{L-1} n\right]+\tau_{2} \cos \left[\frac{4 \pi}{L-1} n\right]-\tau_{3} \cos \left[\frac{6 \pi}{L-1} n\right]+\tau_{4} \cos \left[\frac{8 \pi}{L-1} n\right]
$$

where the coefficients $\tau_{0}, \tau_{1}, \tau_{2}, \tau_{3}$, and $\tau_{4}$ are listed in Table 1 and $L$ is the window size. That is, based on the theory stated previously, and using (2), it is possible to generate new window by convolving the flat-top weighted-windw with itself, which corresponds to multiplication in the time-domain, that is, according to (2), the new window can be generated directly by multiplying $w_{F T W}(n)$ by itself. That is, the new window, $w_{F T F T}(n)$, called as $F T F T$-window, can be described as follows:

Table 1. Flat-top weighted-window coefficients [33]

\begin{tabular}{cc}
\hline Coefficient & Corresponding value \\
\hline$\tau_{0}$ & 0.21557895 \\
$\tau_{1}$ & 0.41663158 \\
$\tau_{2}$ & 0.277263158 \\
$\tau_{3}$ & 0.083578947 \\
$\tau_{4}$ & 0.006947368 \\
\hline
\end{tabular}

$$
\begin{aligned}
& w_{F T F T}(n)=\alpha_{0}+\alpha_{1} \cos (\theta n)+\alpha_{2} \cos (2 \theta n)+\alpha_{3} \cos (3 \theta n)+\alpha_{4} \cos (4 \theta n)+\alpha_{5} \cos (5 \theta n)+ \\
& \alpha_{6} \cos (6 \theta n)+\alpha_{7} \cos (7 \theta n)+\alpha_{8} \cos (8 \theta n)
\end{aligned}
$$

where

$$
\theta=\frac{2 \pi}{L-1}
$$

and $\alpha_{0}, \alpha_{1}, \alpha_{2}, \alpha_{3}, \alpha_{4}, \alpha_{5}, \alpha_{6}, \alpha_{7}$, and $\alpha_{8}$ are listed in Table 2. This window is used to windowing the traditional sinc-fuction to obtain the windowed-sinc filter that is used in this paper.

$$
\psi(n)=\frac{\sin \left(\omega_{c} n\right)}{\omega_{c} n} \cdot w_{F T F T}(n)
$$

where $\omega_{c}$ stands for cut-off frequency. Then F-OFDM signal can be represented as:

Table 2. FTFT-window factors

\begin{tabular}{cc}
\hline Factor & Value \\
\hline$\alpha_{0}$ & 0.17521950295 \\
$\alpha_{1}$ & -0.31890460119 \\
$\alpha_{2}$ & 0.24308301561 \\
$\alpha_{3}$ & -0.15444680377 \\
$\alpha_{4}$ & 0.07924988333 \\
$\alpha_{5}$ & -0.02606785569 \\
$\alpha_{6}$ & 0.00541896938 \\
$\alpha_{7}$ & -0.00058065370 \\
$\alpha_{8}$ & 0.000024132961 \\
\hline
\end{tabular}

Starting with the collection of binary data, the F-OFDM transmitter component viewing begins. By applying $M$-QAM mapping, data is transformed depending on baseband modulation order ( $M$-QAM). Once serial to parallel conversion has taken place, the samples will be available for the $N$-points inverse discrete fourier transform (IDFT) using its fast version inverse fast foureir transform (IFFT). Multiplexing occurs after $N$-IFFT operations, which means the signal is changed from parallel to serial arrangement. Then the filter action will be performed as a last baseband operation, as indicated in Figure 1. Note that the conventional CP-OFDM is identical to one chain of Figure 1 but without the filter. Furthermore, the receiving part of the F-OFDM or the CP-OFDM is the reverse of these discussed procedures in Figure 1.

$$
x(n)=\left[\frac{1}{\sqrt{N}} \sum_{k=0}^{N-1} X_{i}(k) e^{j 2 \pi \frac{k n}{N}}\right] * \psi(n)
$$

in the last expression, $k=0 \ldots N$ is the frequency indes.

Consequently, the simulation results will confirm that the developed windowed-sinc filter in this work will reduce the OOB emission suggestively. Further, the sidelobe of the new window will be significantly clear as will be shown in the next section. 


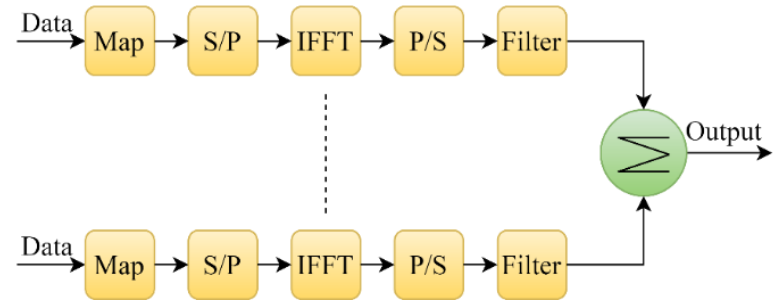

Figure 1. Effects of selecting different switching under dynamic condition

\section{RESULTS AND DISCUSSION}

A comparsion between conventional CP-OFDM and suggested F-OFDM will be conducted in this section. However, the suggested F-OFDM will be built around two other windowed-sinc filters which are Hamming and Hanning based filters. The much more essential simulation parameters are shown in Table 3. As shown in Table 3, an adequate number of Monte-Carlo rounds will be required to even get correct results.

Table 3. Essential simulation parameters

\begin{tabular}{cc}
\hline Paramerers & Settings \\
\hline$N$ & 1024 \\
$L$ & 511 \\
$M$ & 64,256 \\
Number of resource blocks & 50 \\
Number of subcarriers & 12 \\
Tone offset & 2 \\
Monte-Carol rounds & 10,000 \\
\hline
\end{tabular}

A considerable reduction in the OOB emission is required in order to avoid interference in asynchronous communications with variable numerology. It is feasible to integrate the function blocks shown in Figure 1 by using the settings provided in Table 3. That is, the Invers/FFT size, $N$, is set to 1024, the filter length, $L=N / 2-1$, is 511-points [6], [8], [29]. Further, the constellation mapping, $M$, will be 256, in other words, 256-QAM. There will be 50-resource blocks, each one of them includes 12-subchannels. The toneoffset, which is decay-up/down interval is 2 -subcarriers. The simulation will be achieved for ten thousand as Monte-Carlo iterations. Figure 2 illusterates the time-domain representation of the suggested window.

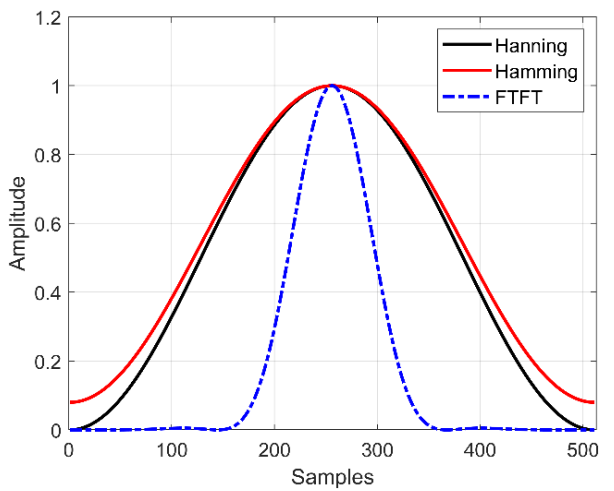

Figure 2. Time-domain plot of the suggested window compared with Hanning and Hamming windows

In Figure 2, it can be seen that the newly developed window, FTFT, has less mainlobe width than the other two windos, Hamming and Hanning windows, which is the required characteristic in the 5G telecommunication systems. On the other hand, the frequency-domain of the suggested window is compared with Hamming and Hanning windows in Figure 3. That is, the spectral density of FTFT-window has the lowest sidelobe compared to Hamming and Hanning windows. In numeric language, the first sidelobe of 
FTFT-window is starts at $-141 \mathrm{dBW} / \mathrm{Hz}$, when compared to Hamming, the first sidelobe appeared at $-61.56 \mathrm{dBW} / \mathrm{Hz}$, while Hanning shows sidelobe at $-56.0 \mathrm{dBW} / \mathrm{Hz}$.

Henceforth, the sidebloes of the produced F-OFDM signals can be seen in Figure 4. That is, the the Hamming-based F-OFDM signal shows lower power spectral density (PSD), $-157.2 \mathrm{dBW} / \mathrm{Hz}$, which is outstanding CP-OFDM-PSD, $-50 \mathrm{dBW} / \mathrm{Hz}$. Moreover, PSD of Hanning-based F-OFDM reduced to $-166.3 \mathrm{dBW} / \mathrm{Hz}$, which is also an enhanced version compared to that of the CP-OFDM signal. However, FTFT-based F-OFDM signal shows lowest PSD which is reached down to $-270.9 \mathrm{dBW} / \mathrm{Hz}$, which is exteremely a very improved signal compared to that of CPOFDM, Hamming-based F-OFDM, and Hanning-based F-OFDM signals. For that reason, the recommended window shows better performance and can be implemented to accommodate for the different types of signals required by different communication devices, such as MTC and ultra reialible low latency communication systems.

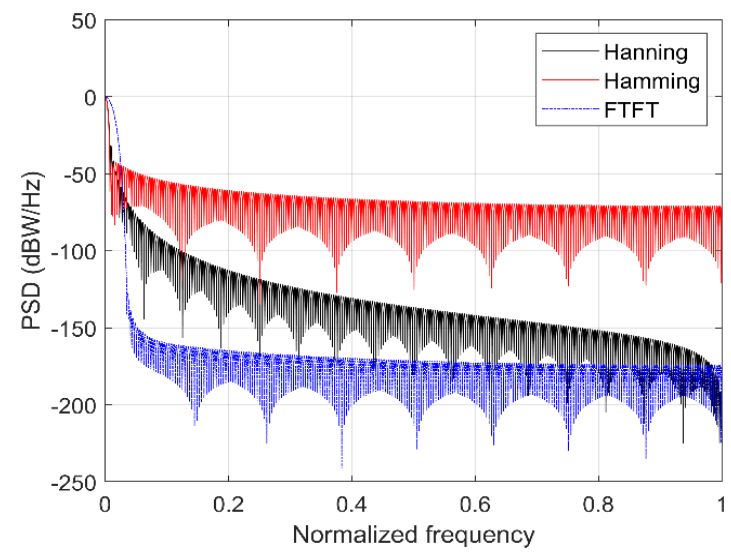

Figure 3. Frequency-domain plot of FTFT-window, hanning-, and hamming-windows

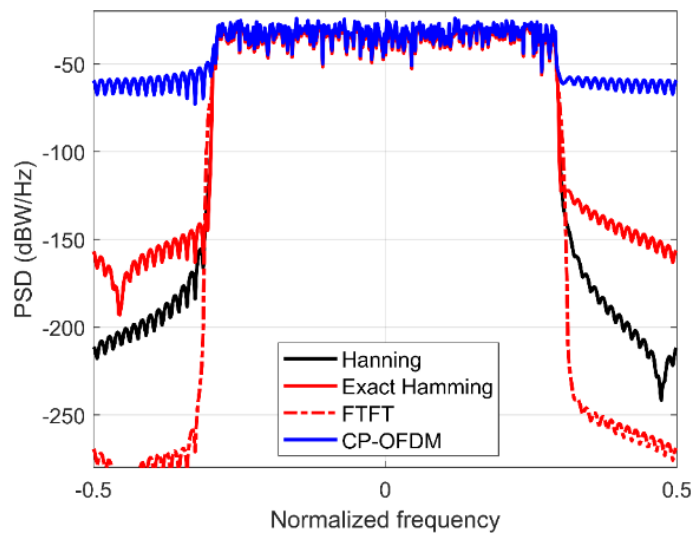

Figure 4. Power spectral density illusteration of CP-OFDM, hanning-, hamming-, and FTFT-based F-OFDM signals

Last but not least, the bit error rate (BER) performance of the four signals, CP-OFDM, Hamming-, Hanning, and FTFT-based F-OFDM signals are compared in Figure 5, which shows that BER act of the suggested FTFT-F-OFDM has no significant difference as compared to the other three signals. In other words, the introduced window has achieved the goal it was designed for. 


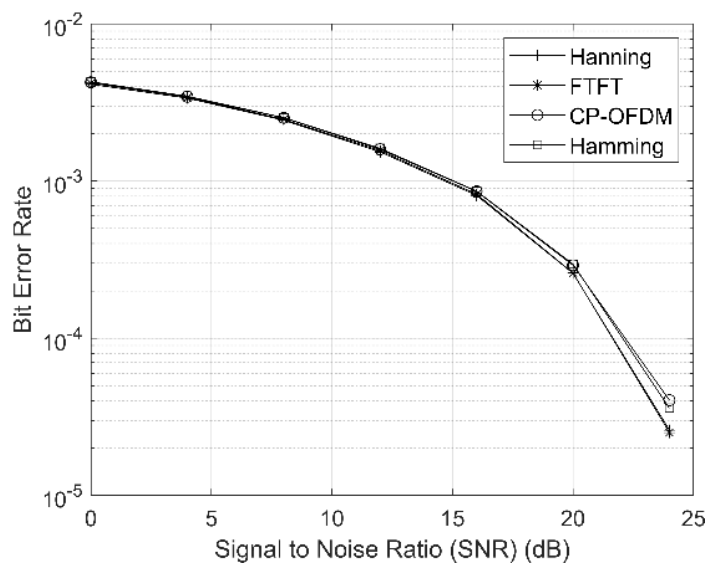

Figure 5. BER performance comparison of CP-OFDM, hanning-, hamming-, and FTFT-based F-OFDM signals

\section{CONCLUSION}

There's a need for synchronization-free communications to better 5G networks with varied numerology needs. In this regard, F-OFDM is a potential contender. Using a new filter architecture, the FOFDM waveform's time-frequency localisation has been enhanced substantially relative to CP-OFDM. Despite increasing complexity, the suggested design may be executed on-line and still preserve the typical FOFDM topology neither the symbol size nor the baseband modulation are restricted in the new model.

\section{REFERENCES}

[1] M. H. Wali, A. K. Jassim, H. M. Almgotir, "Design and analysis 5G mobile network model to enhancement high-density subscribers," Bulletin of Electrical Engineering and Informatics, vol 10, no. 3, pp. 1464-1474, 2021, doi: 10.11591/eei.v10i3.2107.

[2] J. G. Andrews et al., "What Will 5G Be?," in IEEE Journal on Selected Areas in Communications, vol. 32, no. 6, pp. 1065-1082, June 2014, doi: 10.1109/JSAC.2014.2328098.

[3] Shehab, J.N., H.R. Hatem, and O.A. Mahmood, "Hiding (1-8) Multimedia Files In One Color Image," Diyala Journal of Engineering Sciences, vol. 10, no. 3, pp. 54-62, 2017, doi: 10.24237/djes.2017.10305.

[4] P. Banelli, S. Buzzi, G. Colavolpe, A. Modenini, F. Rusek and A. Ugolini, "Modulation Formats and Waveforms for 5G Networks: Who Will Be the Heir of OFDM?: An overview of alternative modulation schemes for improved spectral efficiency," in IEEE Signal Processing Magazine, vol. 31, no. 6, pp. 80-93, Nov. 2014, doi: 10.1109/MSP.2014.2337391.

[5] S. Schwarz, T. Philosof and M. Rupp, "Signal Processing Challenges in Cellular-Assisted Vehicular Communications: Efforts and developments within 3GPP LTE and beyond," in IEEE Signal Processing Magazine, vol. 34, no. 2, pp. 47-59, March 2017, doi: 10.1109/MSP.2016.2637938.

[6] F. Schaich, T. Wild and Y. Chen, "Waveform Contenders for 5G - Suitability for Short Packet and Low Latency Transmissions," 2014 IEEE 79th Vehicular Technology Conference (VTC Spring), 2014, pp. 1-5, doi: 10.1109/VTCSpring.2014.7023145.

[7] M. A. Taher and A. A. Mohammed, "Power Envelope Variation Improvement Of Downlink LTE System Using Complex Number Manipulation Approach,” Diyala Journal of Engineering Sciences, vol. 8, no. 4, pp. 618-623, 2015.

[8] Y. Liu et al., "Waveform Design for 5G Networks: Analysis and Comparison," in IEEE Access, vol. 5, pp. 19282-19292, 2017, doi: 10.1109/ACCESS.2017.2664980.

[9] T. Wild, F. Schaich and Y. Chen, "5G air interface design based on Universal Filtered (UF-)OFDM," 2014 19th International Conference on Digital Signal Processing, 2014, pp. 699-704, doi: 10.1109/ICDSP.2014.6900754.

[10] M. Bellanger et al., "FBMC physical layer : a primer, " PHYDYAS, pp. 1-31, 2010.

[11] N. Michailow et al., "Generalized Frequency Division Multiplexing for 5th Generation Cellular Networks," in IEEE Transactions on Communications, vol. 62, no. 9, pp. 3045-3061, Sept. 2014, doi: 10.1109/TCOMM.2014.2345566.

[12] A. Bedoui and M. Et-tolba, "A comparative analysis of filter bank multicarrier (FBMC) as 5G multiplexing technique," 2017 International Conference on Wireless Networks and Mobile Communications (WINCOM), 2017, pp. 1-7, doi: 10.1109/WINCOM.2017.8238200.

[13] X. Cheng, Y. He, B. Ge and C. He, "A Filtered OFDM Using FIR Filter Based on Window Function Method," 2016 IEEE 83rd Vehicular Technology Conference (VTC Spring), 2016, pp. 1-5, doi: 10.1109/VTCSpring.2016.7504065.

[14] X. Wang, T. Wild, F. Schaich and A. Fonseca dos Santos, "Universal Filtered Multi-Carrier with Leakage-Based Filter Optimization," European Wireless 2014; 20th European Wireless Conference, 2014, pp. 1-5.

[15] S. Lu, D. Qu and Y. He, "Sliding Window Tone Reservation Technique for the Peak-to-Average Power Ratio Reduction of FBMC-OQAM Signals," in IEEE Wireless Communications Letters, vol. 1, no. 4, pp. 268-271, August 2012, doi: 10.1109/WCL.2012.062512.120360.

[16] J. Li, K. Kearney, E. Bala and R. Yang, "A resource block based filtered OFDM scheme and performance comparison," ICT 2013, 2013, pp. 1-5, doi: 10.1109/ICTEL.2013.6632084.

[17] J. Li, E. Bala, and R. Yang, "Resource block Filtered-OFDM for future spectrally agile and power efficient systems," Phys. Commun., vol. 11, pp. 36-55, 2014, doi: https://doi.org/10.1016/j.phycom.2013.10.003. 
[18] Hussain, G.A. and L. Audah, "BCH codes in UFMC: A new contender candidate for 5G communication systems," Bulletin of Electrical Engineering and Informatics, vol. 10, no. 2, pp. 904-910, 2021, doi: 10.11591/eei.v10i2.2080.

[19] X. Zhang, M. Jia, L. Chen, J. Ma and J. Qiu, "Filtered-OFDM - Enabler for Flexible Waveform in the 5th Generation Cellular Networks," 2015 IEEE Global Communications Conference (GLOBECOM), 2015, pp. 1-6, doi: 10.1109/GLOCOM.2015.7417854

[20] J. Abdoli, M. Jia and J. Ma, "Filtered OFDM: A new waveform for future wireless systems," 2015 IEEE 16th International Workshop on Signal Processing Advances in Wireless Communications (SPAWC), 2015, pp. 66-70, doi: 10.1109/SPAWC.2015.7227001.

[21] J. Wang et al., "Spectral Efficiency Improvement With 5G Technologies: Results From Field Tests," in IEEE Journal on Selected Areas in Communications, vol. 35, no. 8, pp. 1867-1875, Aug. 2017, doi: 10.1109/JSAC.2017.2713498.

[22] C. An, B. Kim and H. Ryu, "WF-OFDM (windowing and filtering OFDM) system for the 5G new radio waveform," 2017 IEEE XXIV International Conference on Electronics, Electrical Engineering and Computing (INTERCON), 2017, pp. 1-4, doi: 10.1109/INTERCON.2017.8079635.

[23] "LTE; Evolved Universal Terrestrial Radio Access (E-UTRA); Base Station (BS) radio transmission and reception (3GPP TS 36.104 version 14.3.0 Release 14)," 3GPP LTE 36.104, G.T., 2017.

[24] H. Bölcskei, "Orthogonal Frequency Division Multiplexing Based on Offset QAM," in Advances in Gabor Analysis, pp. 321-352, 2003, doi: 10.1007/978-1-4612-0133-5_12.

[25] J. Bazzi, P. Weitkemper, K. Kusume, A. Benjebbour and Y. Kishiyama, "Design and Performance Tradeoffs of Alternative MultiCarrier Waveforms for 5G,” 2015 IEEE Globecom Workshops (GC Wkshps), 2015, pp. 1-6, doi: 10.1109/GLOCOMW.2015.7414010.

[26] D. Wu et al., "A Field Trial of f-OFDM toward 5G," 2016 IEEE Globecom Workshops (GC Wkshps), 2016, pp. 1-6, doi: 10.1109/GLOCOMW.2016.7848810.

[27] K. C. Hu and A. G. Armada, "SINR analysis of OFDM and f-OFDM for machine type communications," 2016 IEEE 27th Annual International Symposium on Personal, Indoor, and Mobile Radio Communications (PIMRC), 2016, pp. 1-6, doi: 10.1109/PIMRC.2016.7794702.

[28] S. K. Mitra, "Digital signal processing: a computer-based approach,” 2nd ed. New York: McGraw-Hill, 2006.

[29] A. A. Sahrab and A. D. Yaseen, "Filtered orthogonal frequency division multiplexing for improved 5G systems," Bulletin of Electrical Engineering and Informatics, vol. 10, no. 4, pp. 2079-2087, 2021, doi: 10.11591/eei.v10i4.3119

[30] M. A. Taher, H. S. Radhi, and A. K. Jameil, "Enhanced F-OFDM candidate for 5G applications," Journal of Ambient Intelligence and Humanized Computing, vol. 12, pp. 635-652, 2021, doi: 10.1007/s12652-020-02046-3.

[31] A. Antoniou, "Digital signal processing: Signals, systems, and filters," New York: McGraw-Hill, 2006.

[32] F. J. Harris, "On the use of windows for harmonic analysis with the discrete Fourier transform," in Proceedings of the IEEE, vol. 66, no. 1, pp. 51-83, Jan. 1978, doi: 10.1109/PROC.1978.10837.

[33] S. Gade and H. Herlufsen, "Use of weighting functions in DFT/FFT analysis (Part I)," Brüel \& Kjar Technical Review, vol. 3, pp. $1-28,1987$.

\section{BIOGRAPHIES OF AUTHORS}

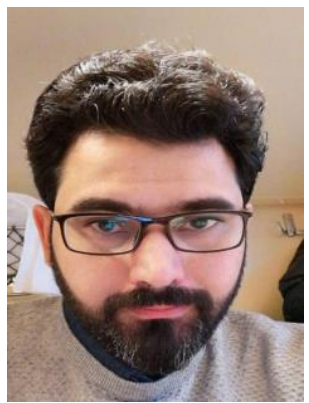

Omar Abdulkareem Mahmood (iD) \&S SC P has received the B.S. degree in communication engineering from College of Engineering, University of Diyala, Diyala, Iraq in 2006, M.Sc. and Ph.D. degrees in electronics and wireless communications from Bonch-Bruevich Saint Petersburg State University of Telecommunications, Saint Petersburg, Russia. He is currently a lecturer with the College of Engineering, University of Diyala. His research interests include telecommunication and networking, digital signal processing and machine learning. He can be contacted at email: omar_abdulkareem@uodiyala.edu.iq.

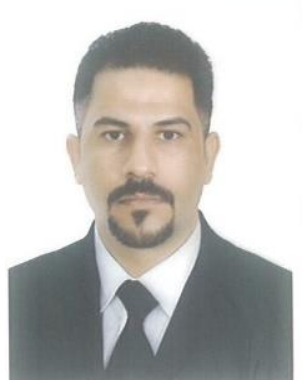

Yousif I. Hammadi (D) SC P was born in Iraq, 1988. He received B.Sc. degree in Communication Engineering from University of Diyala, Iraq, in 2011, and M.Sc. degree in Electronic and Communication Engineering from Cankaya University, Turkey, in 2013. And Ph.D. in Laser/Electronic and Communication Engineering from University of Baghdad, Iraq in 2019. He is working as a Lecturer in Bilad Alrafidain University College, Diyala, Iraq. His research interests include digital communications, optical communication, optical fiber and laser generation. He can be contacted at email: yousif.ibrahim.hammadi@gmail.com. 